\title{
Search for supersymmetry with jets and missing transverse momentum in pp collisions at $13 \mathrm{TeV}$ with CMS
}

\section{Francesco Pandolfi*}

on behalf of the CMS Collaboration

ETH Zurich

E-mail: francesco.pandolfiecern.ch

\begin{abstract}
We present the results of searches for R-parity-conserving Supersymmetry in all-hadronic final states based on a dataset of $12.9 \mathrm{fb}^{-1}$ of $13 \mathrm{TeV}$ proton-proton collisions recorded in 2016 by the CMS experiment at LHC. The results of three searches $\left(H_{\mathrm{T}} / H_{\mathrm{T}}^{\text {miss }}, M_{\mathrm{T} 2}\right.$ and $\left.\alpha_{\mathrm{T}}\right)$ are detailed. The backgrounds to these searches are estimated directly in the data. No significant excess is recorded with respect to the background estimations. Exclusion limits are set, at $95 \%$ confidence level, on simplified models. Depending on the model, gluino masses are excluded up to $1.7 \mathrm{TeV}$, top squarks up to $900 \mathrm{GeV}$, and neutralinos (here considered the lightest Supersymmetric particle) up to $1.2 \mathrm{TeV}$.
\end{abstract}

38th International Conference on High Energy Physics 3-10 August 2016

Chicago, USA

${ }^{*}$ Speaker. 

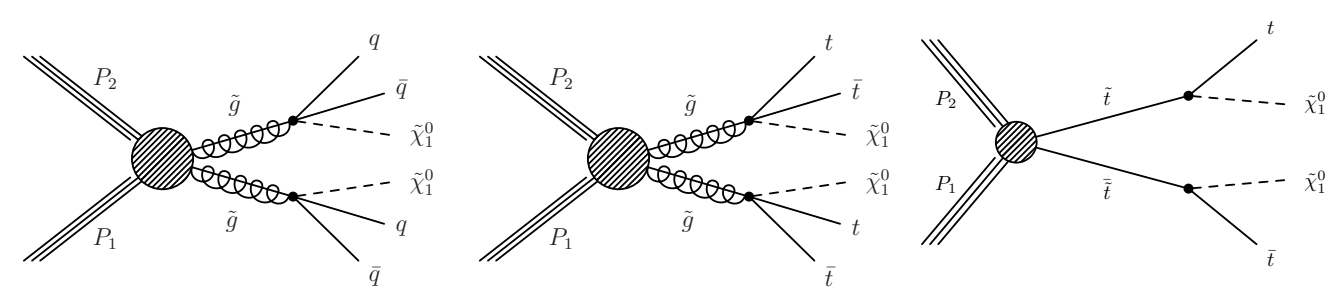

Figure 1: Feynman diagrams of Supersymmetric events relevant for all-hadronic searches. Left: pair production of gluinos, each of which decays to a pair of quarks and the LSP. Middle: pair production of gluinos decaying to pairs of top quarks and LSP. Right: pair production of top squarks.

\section{Introduction}

We present the results of searches for R-parity-conserving Supersymmetry (SUSY) in allhadronic final states based on a dataset of $12.9 \mathrm{fb}^{-1}$ of $13 \mathrm{TeV}$ proton-proton collisions recorded in 2016 by the CMS [1] experiment at LHC. To search for SUSY in all-hadronic final states means to aim for strong production of pairs of gluinos or squarks, exploiting their large production cross section at a hadron collider. In R-parity conserving SUSY, gluinos and squarks are pair-produced, and they decay predominantly in quarks and the lightest supersymmetric particle (LSP), which throughout this report is assumed to be the neutralino $\tilde{\chi}_{1}^{0}$, which is neutral and stable, and goes undetected, like a neutrino.

The signal events that are sought for, therefore, typically present high hadronic activity from the quarks and significant missing transverse energy $\left(E_{\mathrm{T}}^{\mathrm{miss}}\right)$ from the $\tilde{\chi}_{1}^{0}$. Additionally, depending on the signal process, there might be high multiplicity of jets or of jets originating from the hadronization of a bottom quark (b-jets), as can be seen in Figure 1, where Feynman diagrams for typical signal processes are shown. The left diagram shows pair production of gluinos, each of which decays to a pair of light quarks (q) and the LSP: these events will typically present four jets and $E_{\mathrm{T}}^{\text {miss }}$. The central diagram shows the decay of gluinos to pairs of top quarks, with up to 12 jets in the final state, two of which originating from a bottom quark. And finally the third diagram shows pair production of top squarks, which typically results in up to six jets (two of which from a bottom quark) and $E_{\mathrm{T}}^{\mathrm{miss}}$. In order to be sensitive to these different signal decay chains, searches typically employ an event categorization procedure.

CMS employs an event reconstruction technique, known as the Particle Flow, which aims to reconstruct all the particles produced in a proton-proton collision, through the optimal combination of all of its sub-detectors. The resulting list of particle candidates are clustered into jets with the anti- $k_{T}$ algorithm [2], with radius parameter of 0.4 , and are calibrated in situ with di-jet and photon+jet events. Missing transverse energy is defined as the negative vector sum of all the particles in the event. The same calibration applied to jets is also used to correct the $E_{\mathrm{T}}^{\mathrm{miss}}$. The transverse hadronic energy $H_{\mathrm{T}}$ is defined as the scalar sum of the transverse momenta of all jets in the event, and the missing transverse hadronic energy $H_{\mathrm{T}}^{\text {miss }}$ is defined as the negative vectorial sum of all jets in the event. Jets originating from bottom quarks (b-jets) are identified by the combined secondary vertex algorithm [3].

Historically, CMS has performed four searches for SUSY in all-hadronic final states: a search based on $H_{\mathrm{T}}$ and $H_{\mathrm{T}}^{\mathrm{miss}}$ [4], one based on the $M_{\mathrm{T} 2}$ variable [5], one based on $\alpha_{\mathrm{T}}$ [6], and finally 

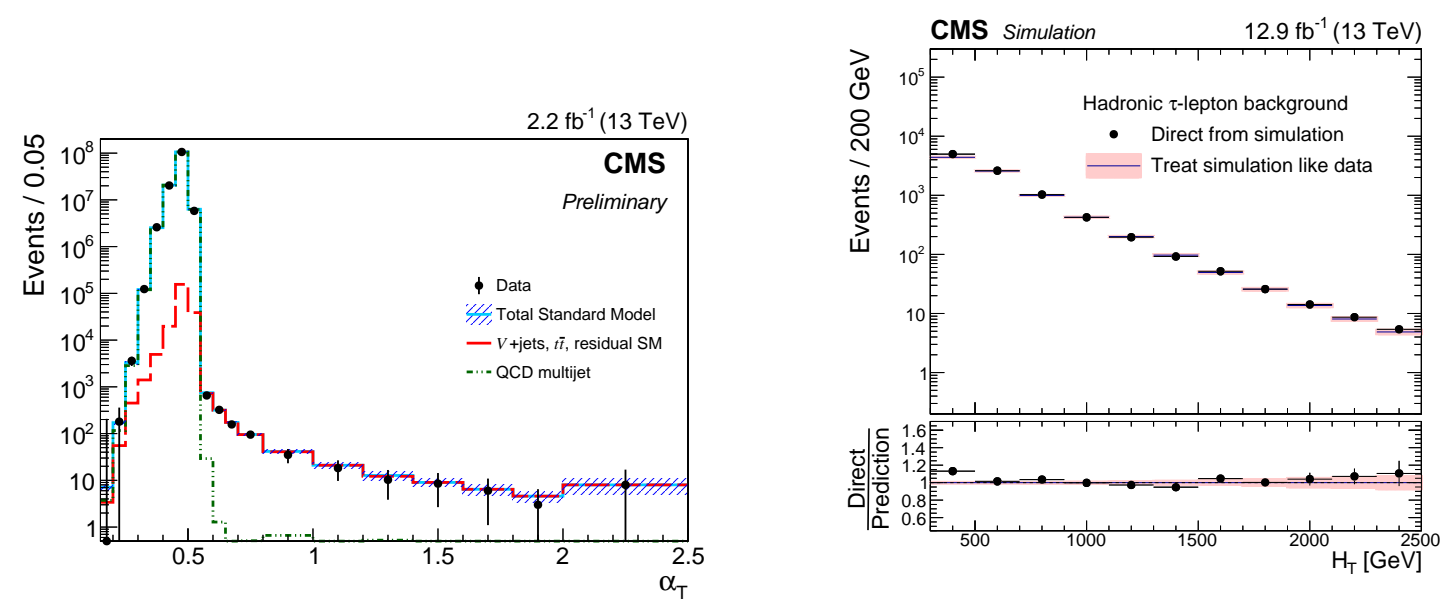

Figure 2: Left: $\alpha_{\mathrm{T}}$ distribution in data (black markers) compared to the expected distribution of the backgrounds: the distribution for QCD events is represented by the hashed green line, while the other backgrounds by a red line; the sum of all background contributions is shown with a dashed cyan line. Right: $H_{\mathrm{T}}$ distribution for simulated events with $W \rightarrow \tau \nu_{\tau}$ decays (black markers) compared with the results of the muon-smearing method applied to the simulation (black lines with pink bands).

one based on Razor variables [7]. The first three searches have updated their results to $12.9 \mathrm{fb}^{-1}$ of data, and therefore are the focus of this talk.

All three analyses employ a veto on events containing leptons and then search for events with high hadronic activity and significant $E_{\mathrm{T}}^{\text {miss }}$. The $H_{\mathrm{T}} / H_{\mathrm{T}}^{\text {miss }}$ analysis is binned in sixteen categories based on jet and b-jet multiplicities, and in each category it is further subdivided with rectangular cuts on $H_{\mathrm{T}}$ and $H_{\mathrm{T}}^{\text {miss }}$. The $M_{\mathrm{T} 2}$ analysis is based on the $M_{\mathrm{T} 2}$ (or 'stranverse mass') variable, which is a missing-energy-like variable, optimized for the case of events with two symmetric decay chains, each ending in an undetected particle; it also categorizes events based on their jet multiplicity, their b-jet multiplicity, and their $H_{\mathrm{T}}$, and in each category it scans the tail of $M_{\mathrm{T} 2}$. Finally, the $\alpha_{\mathrm{T}}$ search is based on the use of the $\alpha_{\mathrm{T}}$ variable at trigger and preselection level; it is also binned in $H_{\mathrm{T}}$ and in jet and b-jet multiplicities, and adopts $H_{\mathrm{T}}^{\text {miss }}$ as search variable.

\section{Background estimation}

Three main Standard Model processes can produce events with large hadronic activity and significant $E_{\mathrm{T}}^{\text {miss }}$, therefore constituting a background for all-hadronic SUSY searches: (i) QCD multijet events, in which the $E_{\mathrm{T}}^{\text {miss }}$ is instrumental, typically coming from the mismeasurement of the energy of one of the jets; (ii) events with a leptonic $\mathrm{W}$ boson decay, where the $E_{\mathrm{T}}^{\text {miss }}$ comes from the neutrino and the charged lepton is not identified; (iii) events with a $\mathrm{Z}$ boson produced in association with jets, and the $\mathrm{Z}$ decays to neutrinos.

While the QCD background has the highest cross section, it is not a major background for these searches. This is because most of the search variables that are employed are effective 'QCDkillers'. This is seen for example in Figure 2 (left) where the $\alpha_{\mathrm{T}}$ distribution is shown: QCD events present a sharp cut-off at around 0.5 , therefore a cut on this variable will already suppress most of this background. Further suppression can be achieved by looking at the angular direction 

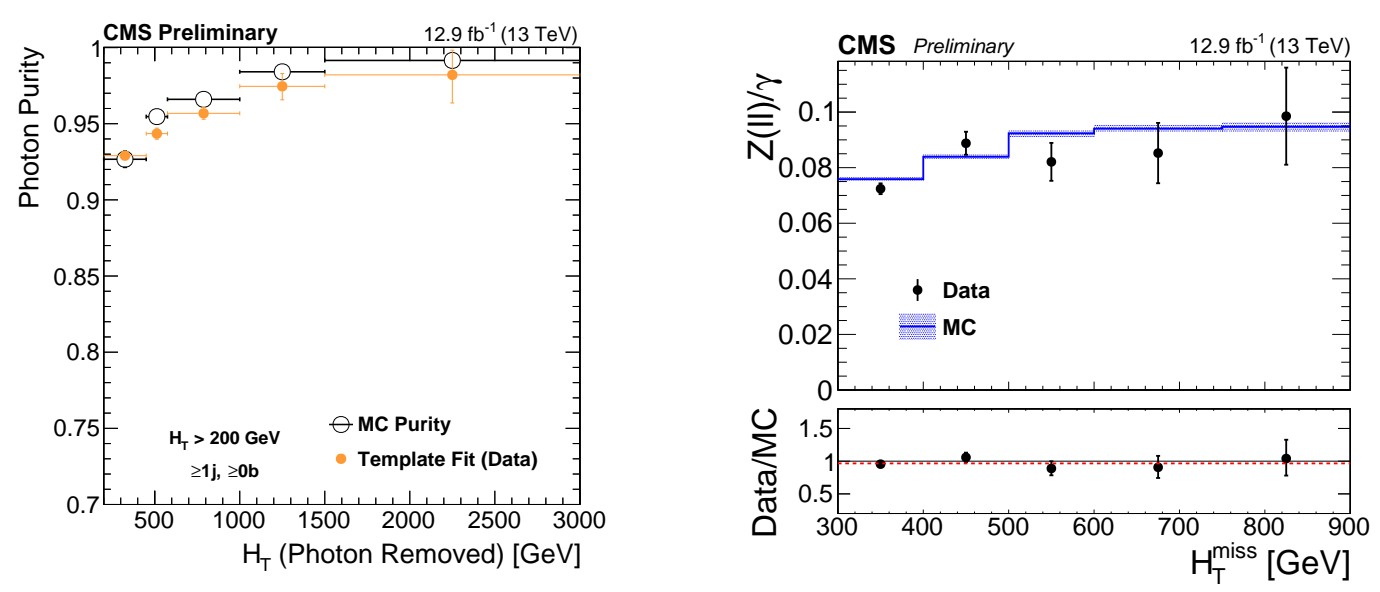

Figure 3: Right: results of the photon purity measurement in data (orange markers) compared to the true purity of simulated events (hollow markers) as a function of $H_{\mathrm{T}}$. Right: $\left(\mathrm{Z} \rightarrow \ell^{+} \ell^{-}\right) / \gamma$ ratio as a function of $H_{\mathrm{T}}^{\text {miss }}$ for data (black markers) and the simulation (blue line); the bottom pad shows the ratio between data and the simulation.

of the $E_{\mathrm{T}}^{\text {miss: }}$ in these events the source of missing energy is instrumental, typically due to the mismeasurement of an energetic jet, so requiring that the $E_{\mathrm{T}}^{\text {miss }}$ vector is not aligned with one of the leading jets in the event is another powerful handle against this background.

Events with a leptonically decaying $\mathrm{W}$ boson have genuine $E_{\mathrm{T}}^{\text {miss }}$ from the neutrino and can constitute a background to these searches if the charged lepton is not identified. The main handle to constrast them is to employ a tight lepton veto. Some events might fail to be vetoed if the charged lepton is 'lost': this can happen if the lepton is produced outside of the detector acceptance, or if it fails to be identified because of the presence of a nearby jet. The residual contribution of this background is therefore evaluated by defining a control region in data with exactly one reconstructed electron or muon, and these events are then multiplied by the probability of 'losing' the lepton, which is taken from the simulation.

The $H_{\mathrm{T}} / H_{\mathrm{T}}^{\text {miss }}$ analysis employs a dedicated treatment for $\mathrm{W} \rightarrow \tau \nu_{\tau}$ events in which the $\tau$ lepton decays hadronically. These are evaluated by taking $\mathrm{W} \rightarrow \mu v_{\mu}$ events in data, and simulating the presence of the $\tau$ by smearing the muon $p_{\mathrm{T}}$ with response functions, taken from the simulation. The method proves to have good closure on the simulation, as is shown in Figure 2 (right), where the $H_{\mathrm{T}}$ distribution for events with $\mathrm{W} \rightarrow \tau \nu_{\tau}$ events (black markers) is compared with the results of the muon-smearing method (black lines with pink bands).

Events with a $\mathrm{Z}$ boson decaying to neutrinos, produced in association with jets, constitute the main irreducible background to these searches. This background is typically estimated with photon+jet events, in which the photon is removed from the event and added to the missing energy in order to simulate the presence of the 'invisible' Z boson decay. One needs to take care of the fact that the photon sample is not completely background-free, because of electromagneticallydecaying mesons that can simulate the signal of a prompt photon. This is done with a purity fit to photon shower shape or isolation variables, which are capable of discriminating between prompt photons and photons produced within the hadronization of a coloured parton. The result of a purity measurement, as a function of $H_{\mathrm{T}}$ is shown in Figure 3 (left) where the purity measured in 


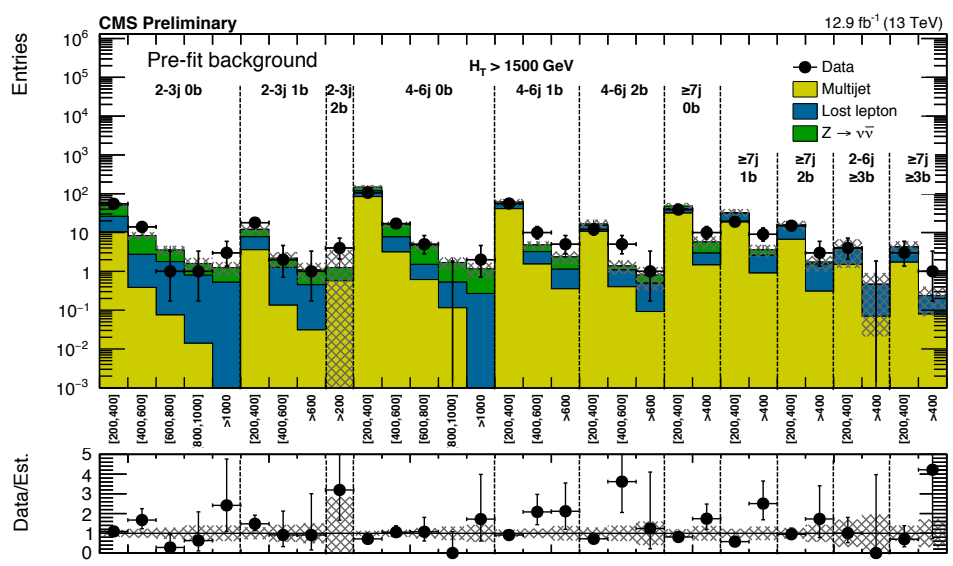

Figure 4: Results of the search in the $H_{\mathrm{T}}>1500 \mathrm{GeV}$ regions of the $M_{\mathrm{T} 2}$ analysis. Each bin corresponds to a different search region. Regions are grouped depending on their jet and b-jet multiplicity, where the $M_{\mathrm{T} 2}$ variable is scanned in increasing intervals (visible on the $\mathrm{x}$-axis label, in $\mathrm{GeV}$ ). The data (black markers) are compared to the stack of the background estimates: QCD multijets (yellow), 'lost' lepton from W decays (blue) and 'invisible' $\mathrm{Z}$ decays (green). The bottom pad shows the ratio between the data and the sum of the background estimates.

data (orange markers) is compared to the one predicted by the simulation (hollow markers). After the QCD background is accounted for, a transfer factor needs to be applied to these events to take into account the different masses and couplings of the bosons. This transfer factor is taken from the simulation but is validated in data with $\mathrm{Z} \rightarrow \ell^{+} \ell^{-}$events (where $\ell=\mathrm{e}$ or $\mu$ ). This validation can be seen in Figure 3 (right) where the $\left(\mathrm{Z} \rightarrow \ell^{+} \ell^{-}\right) / \gamma$ ratio is shown as a function of $H_{\mathrm{T}}^{\text {miss }}$ for data (black markers) and the simulation (blue line).

\section{Results and interpretation}

Once the background estimation techniques are defined, one can compare the data to the sum of the estimates in each analysis search region. An example of this can be seen in Figure 4, where the results of the search in the regions with $H_{\mathrm{T}}>1500 \mathrm{GeV}$ of the $M_{\mathrm{T} 2}$ analysis. Here each bin is a different search region (depending on the jet and b-jet multiplicities, and on the value of $M_{\mathrm{T} 2}$ ) and the data (black markers) are compared to the background estimates: the QCD multijet background is shown in yellow, the $\mathrm{W}$ background with 'lost' charged lepton in blue, and the $\mathrm{Z} \rightarrow v \bar{v}$ background in green. The bottom pad shows the ratio between the data and the sum of the background estimates. No significant excess is observed in the data in any of the searches.

With the use of simplified models, 95\%-confidence-level upper limits on the signal cross section can be set in the plane defined by the mass of the produced particle and the lightest supersymmetric particle $\tilde{\chi}_{1}^{0}$. These limits are derived with a modified frequentist approach $[8,9,10]$ and are shown in Figure 5 for pair production of gluinos, each decaying to b $\bar{b} \tilde{\chi}_{1}^{0}$ (left), $\bar{t} \bar{\chi} \tilde{\chi}_{1}^{0}$ (center) or $\mathrm{q} \overline{\mathrm{q}} \tilde{\chi}_{1}^{0}$ (right). The $H_{\mathrm{T}} / H_{\mathrm{T}}^{\text {miss }}$ results are shown in blue, the $M_{\mathrm{T} 2}$ results in yellow, and the $\alpha_{\mathrm{T}}$ results in green. In the center plot the results of different searches are also shown. In all plots the expected limits are shown as dotted lines and the observed ones as solid lines. As can be seen gluino masses of up to $1.75 \mathrm{TeV}$ and $\tilde{\chi}_{1}^{0}$ masses up to $1.2 \mathrm{TeV}$ are excluded. 

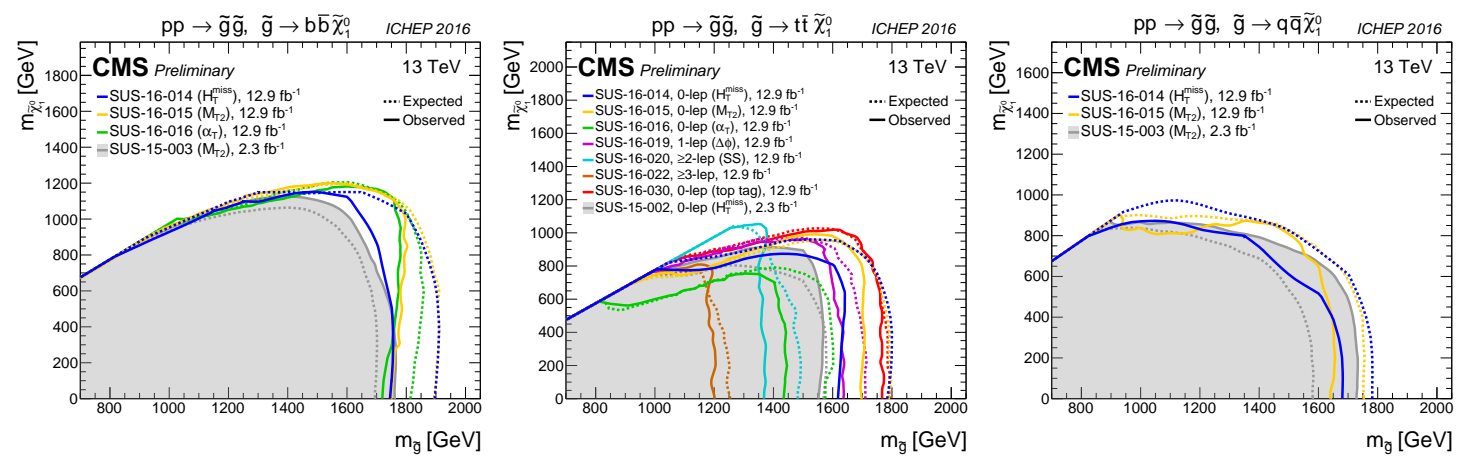

Figure 5: Exclusion limits at 95\% CL on the cross sections of gluino pair production, each decaying to $\mathrm{b} \bar{b} \tilde{\chi}_{1}^{0}$ (left), $\mathrm{t} \bar{\chi} \tilde{\chi}_{1}^{0}$ (center) or $\mathrm{q} \bar{q} \tilde{\chi}_{1}^{0}$ (right), in the plane defined by the gluino and the $\tilde{\chi}_{1}^{0}$ mass. The area to the left of and below the thick black curve represents the observed exclusion region, while the dashed line represent the expected limits. The results of the $H_{\mathrm{T}} / H_{\mathrm{T}}^{\text {miss }}$ search are shown in blue, those of $M_{\mathrm{T} 2}$ in yellow, and those of $\alpha_{\mathrm{T}}$ in green.
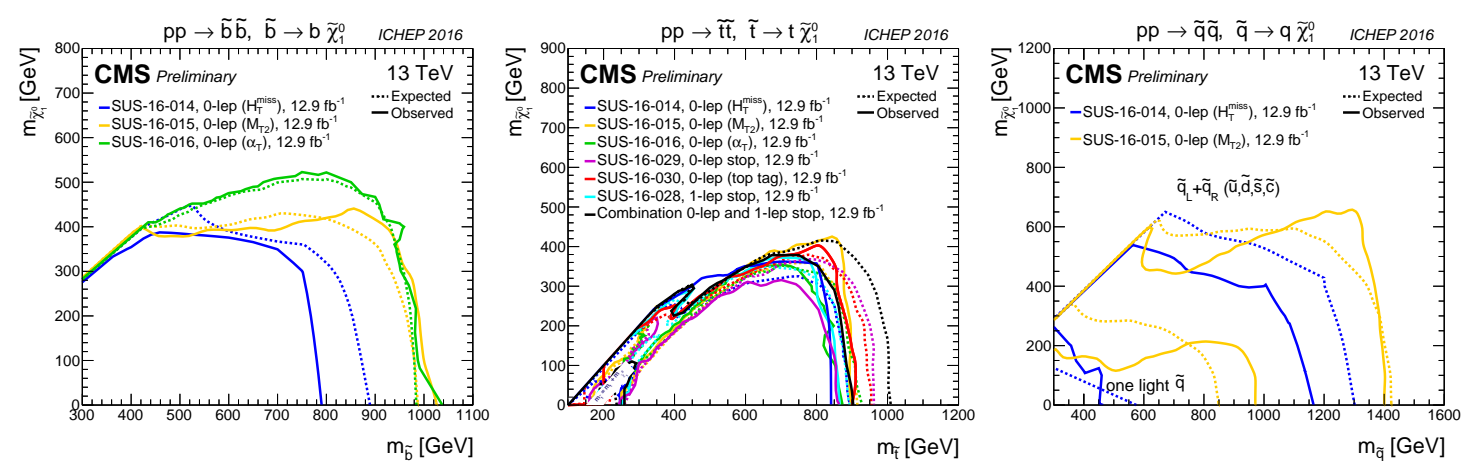

Figure 6: Exclusion limits at $95 \% \mathrm{CL}$ on the cross sections of bottom squark (left), top squark (center) and light squark (right) pair production in the plane defined by the squark and the $\tilde{\chi}_{1}^{0}$ mass. The area to the left of and below the thick black curve represents the observed exclusion region, while the dashed line represent the expected limits. The results of the $H_{\mathrm{T}} / H_{\mathrm{T}}^{\text {miss }}$ search are shown in blue, those of $M_{\mathrm{T} 2}$ in yellow, and those of $\alpha_{\mathrm{T}}$ in green.

Finally, the exclusions for pair production of squarks are shown in Figure 6: the right plot for bottom squark production, the center plot for top squark production, the right plot for light squark production. As can be seen, light squarks masses are excluded up to $1.4 \mathrm{TeV}$, top squarks up to $900 \mathrm{GeV}$, and neutralinos up to $500 \mathrm{GeV}$. In the center plot results from other searches are also shown: for details please refer to the contribution by N. Strobbe.

\section{Conclusions}

In summary, we have presented the results of the search for SUSY in all-hadronic final states performed by CMS on a total of $12.9 \mathrm{fb}^{-1}$ of $13 \mathrm{TeV}$ proton-proton collisions delivered by LHC in 2016. No significant excess has been observed, and limits have been set, at $95 \%$ confidence level, on simplified models. 


\section{References}

[1] CMS Collaboration, The CMS experiment at the CERN LHC, JINST 3 (2008) S08004, doi:10.1088/1748-0221/3/08/S08004

[2] M. Cacciari, G. P. Salam, and G. Soyez, The anti- $k_{T}$ jet clustering algorithm, JHEP 04 (2008) 063, doi:10.1088/1126-6708/2008/04/063, arXiv:0802.1189.

[3] CMS Collaboration, Performance of b-tagging algorithms in 25 ns data at $13 \mathrm{TeV}$, CMS Detector Performance Summary CMS-DP-2015-056, 2015

[4] CMS Collaboration, Search for supersymmetry in events with jets and missing transverse momentum in proton-proton collisions at $13 \mathrm{TeV}$, CMS-PAS-SUS-16-014, cds.cern.ch/record/2205158

[5] CMS Collaboration, Search for new physics in the all-hadronic final state with the $M_{\mathrm{T} 2}$ variable, CMS-PAS-SUS-16-015, cds.cern.ch/record/2205162

[6] CMS Collaboration, An inclusive search for new phenomena in final states with one or more jets and missing transverse momentum at $13 \mathrm{TeV}$ with the $\alpha_{\mathrm{T}}$ variable, CMS-PAS-SUS-16-016, cds.cern.ch/record/2205163

[7] CMS Collaboration, Inclusive search for supersymmetry using the razor variables at sqrt(s) $=13 \mathrm{TeV}$, CMS-PAS-SUS-15-004, cds.cern.ch/record/2114815

[8] A. L. Read, Presentation of search results: The CLs technique, J. Phys. G 28 (2002) 2693, doi:10.1088/0954-3899/28/10/313.

[9] A. L. Read, Modified frequentist analysis of search results (The CLs method), CERN-OPEN 205 (2000).

[10] G. Cowan, K. Cranmer, E. Gross, and O. Vitells, Asymptotic formulae for likelihood-based tests of new physics, Eur. Phys. J. C 71 (2011) 1554, doi:10.1140/epjc/s10052-011-1554-0, arXiv:1007.1727. 